\title{
Intramasseteric Cavernous Haemangioma: A Case Report.
}

\author{
N. Charaihenba ${ }^{1}$, T. Rebecca ${ }^{2}$, N. Jiten ${ }^{3}$, Genom Pangkam $^{4}$ \\ ${ }^{1,2,3,4}$ (Ent Department,Rims, Imphal,India)
}

\begin{abstract}
Intramuscular haemangiomas (IMHs) of masseter muscle are extremely rare in head and neck region and often misdiagnosed as parotid tumours. We report a case of a 40 yrs old female who presented with a progressive non tender swelling onright side of face for more than 12 months. MRI and Doppler USG of parotid region revealed a heterogeneous highly vascularized mass located deep to masseter muscle. Patient underwent surgical resection viaretrograde tracing of facial nerve branches and without need for superficial parotidectomy. Pathological examination of resected mass revealed a cavernousIMH.
\end{abstract}

Keywords: Intramuscular haemangioma, masseter muscle, superficial parotidectomy.

\section{Introduction}

Intramuscular haemangioma (IMH) of head and neckregion is extremely rare, accounting for $<1 \%$ of all cases ofhaemangioma ${ }^{1}$.In head and neckregion, masseter muscle is most commonly involved followed by trapezius and sternocleidomastoid muscles. They generally present as gradually enlarging lesions leading to cosmetic problems ${ }^{2}$.Due to the location and characteristics of intramasseteric IMHs, it is difficult to make a correct diagnosis prior to surgery ${ }^{3}$.Here we report an intramasseteric IMH in a 40 yrs old female who underwent excision via modified Blair's incision and retrograde tracing of facial nerve branches without the need for additional superficial parotidectomy and we reviewed the available literature regarding the clinical radiology, pathology and treatment.

\section{Case Report}

A 40- year-old female with no previous significant medical history presented with a painless mass on right side of face for 12 months at department of Otorhinolaryngology, Regional Institute of Medical Sciences, Imphal. Physical examination revealed a $4 \mathrm{~cm} \times 3 \mathrm{~cm}$ mass in the right cheek, mobile anteroposteriorly, located just above the angle of mandible. The mass became firmer and immobile upon clenching teeth (Fig 1). Rest of otorhinolaryngological examination was normal. MR imaging showed a well defined, homogenously enhancing lesion $(4.3 \mathrm{~cm} \times 2.3 \mathrm{~cm} \times 3.1 \mathrm{~cm})$ in the right masticator space overlying cortex of the ramus of the mandible deep to the masseter, hypointense on T1 and hyperintense on T2. (Fig.2). Doppler ultrasonography showed heterogeneous echotexture and hypoechoic peripheral as well as intralesional vascular pattern with no definite draining and feeding artery.Fine needle aspiration of the mass was suggestive of vascular lesion. Patient underwent excision of the mass under general anaesthesia.Modified Blair's skin incision was given and skin flap raised till the anterior border of the masseter muscle. Retrograde tracing of the branches of the facial nerve was done and branches preserved(Fig.3,4,5) The tumour along with a cuff of surrounding masseter muscle was dissected out.After haemostasis and placing of the drainage tube, wound was closed in layers. The drainage tube was removed two days after surgery (Fig 6). Histopathology of the excised specimen revealed cavernous haemangioma (Fig.7). Postoperative period was uneventful.

\section{Discussion}

In the head and neck region, IMHs are most frequently seen in the masseter muscle followed by trapezius, periorbital, sternocleidomastoid, and temporalis muscles in frequency. The tongue, extra-ocular, and posterior neck muscles have also been reported to be involved with haemangioma, but with less frequency ${ }^{4}$.

IMHs generally present as progressively enlarging and often painful lesions. Because of their deep location, they rarely display any clinical signs or symptoms that suggest a vascular nature, such as pulsations, thrills, or bruits. Overlying skin discoloration is also uncommon. The absence of pathognomonic clinical findings and the rare incidence of these lesions make accurate pre-operative diagnosis difficult ${ }^{2,5}$ and only $8 \%$ of all cases of IMHs are diagnosed before surgical intervention.A variety of muscle neoplasms, benign muscular hypertrophy, congenital cysts, and lymphadenopathies are commonly confused in differential diagnosis 5 .

Histologically, the lesions are classified as capillary (vessels smaller than 140 micrometers in diameter), cavernous (vessels larger than 140 micrometers in diameter), or mixed ${ }^{6}$. Capillary haemangioma is the most frequent haemangioma, located in the cutaneous or subcutaneous tissues and diagnosed in the first decade of life. Most instances of capillary haemangioma undergo involution spontaneously. Cavernous haemangiomas are large and deeply located and are diagnosed later in life; these lesions are frequently 
intramuscular, do not have spontaneous involution, and require surgical treatment. Mixed haemangioma is, microscopically, a mixture of capillary and cavernous hemangioma ${ }^{7}$.

Preoperatively, IMHs on plain radiography occasionally demonstrates phleboliths within the lesion. In ultrasound examinations, heterogeneous, echogenic, cystic mass with posterior acoustic shadowing secondary to the calcified nodular areas is often revealed ${ }^{4,6}$. With non-enhanced CT, an ill-defined mass of similar attenuation to muscle may be identified. Phleboliths too small to identify on radiographs can be revealed. After administration of contrast material, significant enhancement is typical ${ }^{7}$. Currently, MRI is the standard imaging technique for diagnosing soft tissue haemangioma ${ }^{7}$. On T1-weighted images, compared with muscle tissue, intensities of the lesions are isointense or hyperintense with unclear margins ${ }^{8}$.On T2-weighted images, haemangiomas are typically hyperintense and have clear margins and lobulated contours ${ }^{8}$. In our case, MRI findings were identical with the literature and have characteristic signs for haemangioma-like high signal intensity on T2-weighted MRI images.

Management of IMHs should be individualized according to the size and anatomic accessibility of the tumour, its growth rate, age of the patient, and cosmetic and functional considerations. Many forms of therapy have been suggested, including steroid injections, radiation therapy, injection of sclerosing agents, cryotherapy, and electrocoagulation. However, the optimal management is surgical resection with wide margins of surrounding normal muscle because of the infiltrative nature of IMHs ${ }^{9}$.

Surgery to remove an IMH in the head and neck region may be approached transorally or via superficial parotidectomy with a preauricular incision ${ }^{6}$. Superficial parotidectomy with a preauricular incision removes the tumour completely and preserves the facial nerve with minimal damage, and thus it should be performed when tumours proximal to the parotid gland require resection. Previous studies have also reported that parotidectomy is a safer technique for the removal of rare tumours located in the head and neck region compared with a transoral approach. Additionally, this type of surgery reduces the risk of recurrence ${ }^{6,7}$. In our case, both superficial parotidectomy and transoral approaches were avoided during resection of the tumour along with a cuff of surrounding masseter muscle.

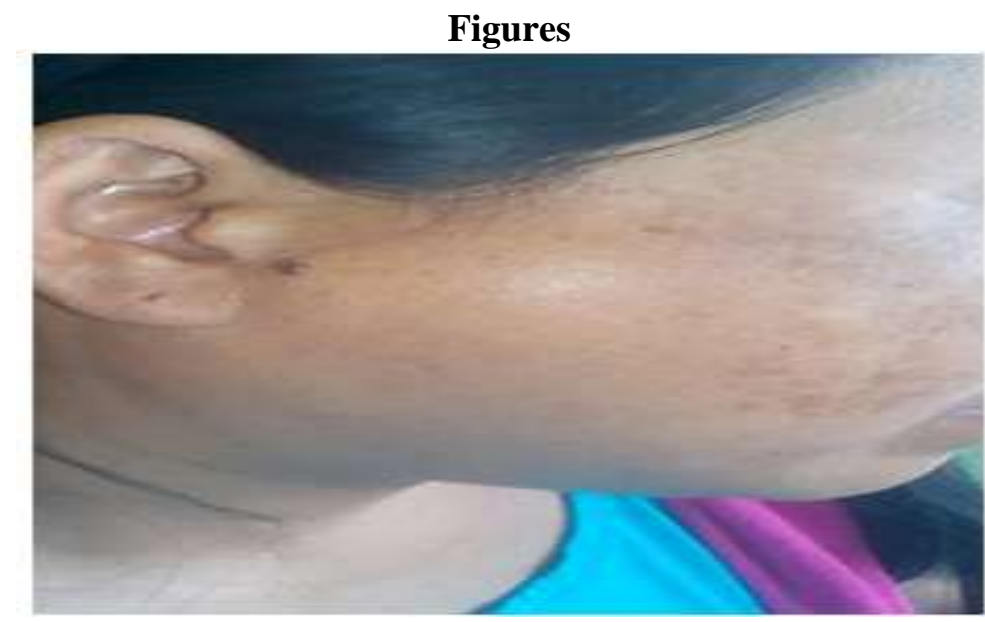

Fig 1. Pre-operative showing facial swelling

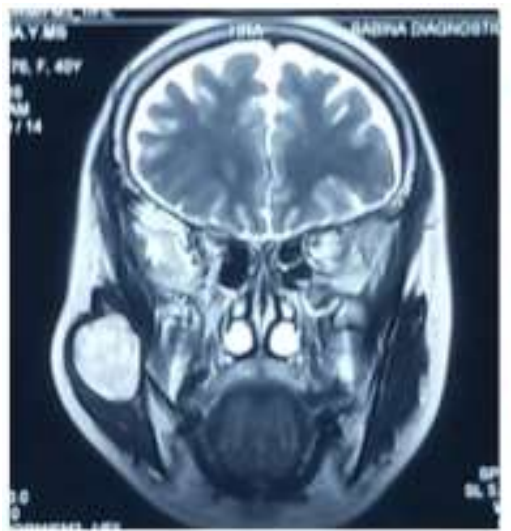

Fig 2: MRI showing the lesion

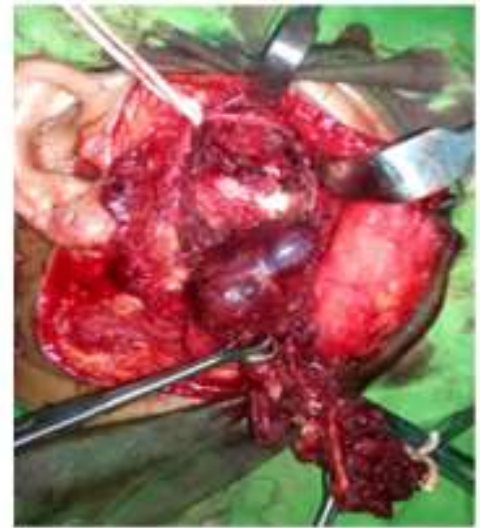

Fig3:intraoperative showing lesion 


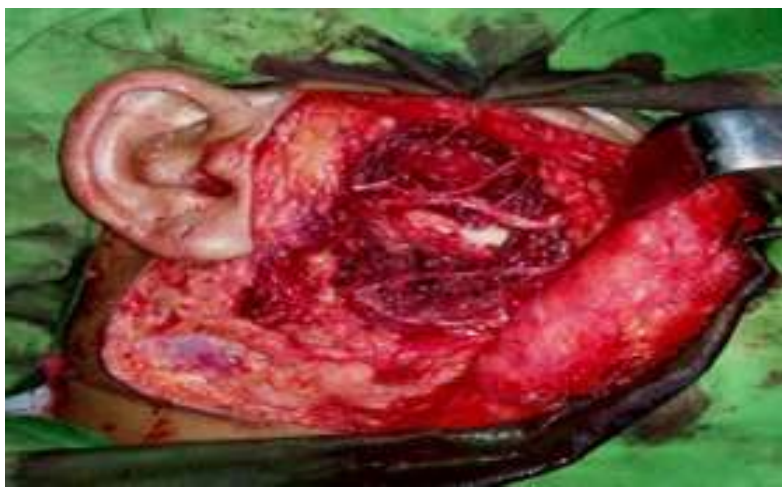

Fig 4: branches of facial nerves

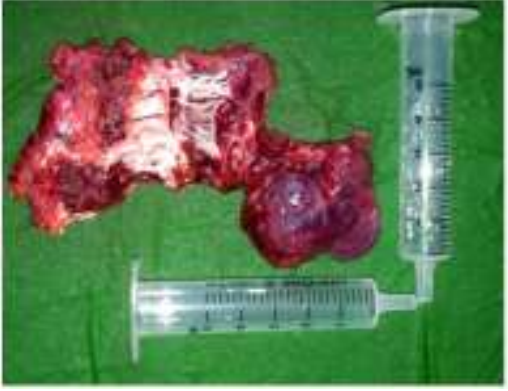

Fig 5: Excision specimen

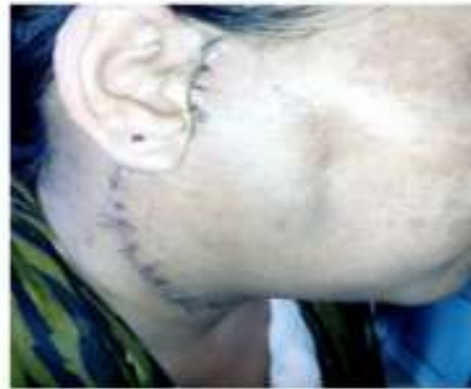

Fig 6: Post operative 5 days

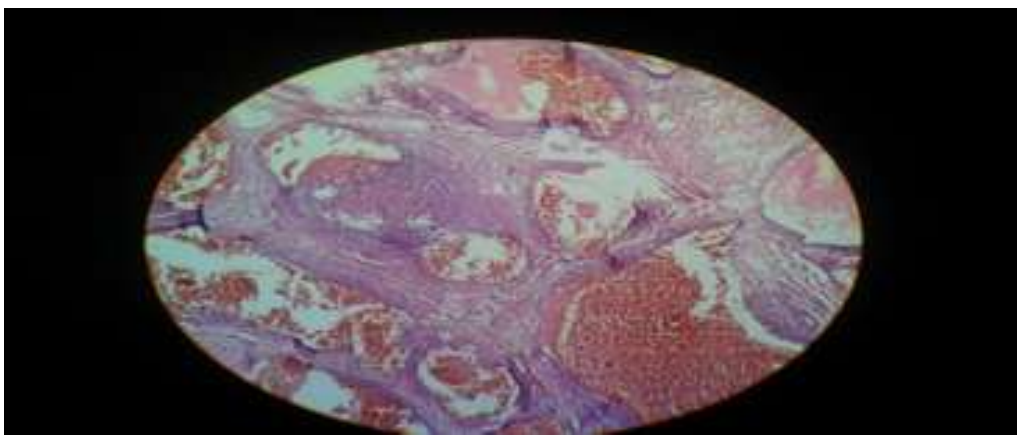

Fig 7: HPE slides suggesting Cavernous Haemangioma

\section{Conclusion}

Intramasseteric haemangioma is rare tumour often can be mistaken for other tumours especially parotid tumours. Preoperative MRI is the best available investigation. In resectable tumours, complete surgical resection is the curative treatment of choice.

\section{References}

[1]. Watson WL and McCarthy WD, Blood and lymph vessel tumours, a report of 1056 cases, Surg Gynecol and Obstet,71,1940,569-588.

[2]. Narayanan CD, Prakash P and Dhanasekaran CK, Intramuscular haemangioma of the masseter muscle, a case report, Cases J 2009, 7459-7460.

[3]. Zengin AZ, Celenka P and Sumer AP, Intramuscular haemangioma presenting with multiple phleboliths, a case report, Oral Surg Oral Med Oral Pathol Oral Radiol, 15, 2013, 32-36.

[4]. Rossiter JL, Hendrix RA, Tom LW and Potsic WP, Intramuscular haemangiomas of the head and neck, Otolaryngol Head Neck Surg 108, 1993, 18-26.

[5]. Demir Z, Oktem F and Celebioglu S, Rare case of intramasseteric cavernous haemangioma in a three-year-old boy, a diagnostic dilemma, Ann Otol Rhino Laryngol, 113, 2004, 455-458.

[6]. Wolf GT, Daniel F, Arbor A and Krause CJ, Intramuscular haemangioma of the head and neck, Laryngoscope, 95, 1985,210-213.

[7]. Olsen KI, Stacy GS and Montag A, Soft-tissue cavernous haemangioma,Radiographics, 24, 2004, 849-854.

[8]. Vilanova JC, Smirniotopoulos JG, Perez-Andres R et al, Haemangioma from head to toe, MR imaging with pathologic correlation, Radiographics, 24, 2004, 367-3s85.

[9]. Ichimura K, Nibu K and Tanaka T, Essentials of surgical treatment for intramasseteric haemangioma, Eur Arch Otorhinolaryngol $252,1995,125-129$. 